\title{
Painful brachial plexopathy: an unusual presentation of polyarteritis nodosa
}

\author{
S. G. Allan* \\ M.R.C.P. \\ C. C. SMITH* \\ F.R.C.P.
}

H. M. A. TowLA*

B. Med. Biol., M.B.

A. W. DownIE*

F.R.C.P.

\author{
J. C. ClaAk $\dagger$ \\ M.B., Ch.B. \\ * Department of Medicine, The Royal Infirmary, Foresterhill, Aberdeen \\ $\dagger$ Department of Pathology, University of Aberdeen, Foresterhill
}

\begin{abstract}
Summary
An elderly man was admitted to hospital with an unusual painful bilateral brachial neuropathy, which progressed in spite of high dose corticosteroid therapy. Polyarteritis nodosa, characterized by widespread arteriolar involvement, was shown at post-mortem.
\end{abstract}

\section{Introduction}

The clinical presentations of polyarteritis nodosa are classically diverse, reflecting variable vascular involvement of many organs and tissues. Mononeuritis multiplex is the most common neurological sequel (Ford and Siekert, 1965), but asymmetrical polyneuropathies, predominantly involving the lower limbs, are well recognized. Central nervous system involvement is described, but unusual. The syndrome of a progressive, painful, bilateral brachial neuropathy with paralysed upper limbs, and hoarseness due to recurrent laryngeal nerve involvement is unusual and reported now.

\section{Case report}

A 78-year-old retired farm worker was admitted with a one week history of progressive bilateral arm weakness, numbness below the elbows and pain in his shoulder muscles. He had felt generally unwell for 6 weeks and had noticed discomfort in his neck and several ribs. For 2 days he had been hoarse, but denied symptoms of recent intercurrent infection although he had been breathless and wheezy for 10 months. He had not smoked for many years, and had received only bronchodilator and diuretic therapy.

On examination he was lucid, apyrexial and hoarse. His arms dangled helplessly by his sides, but he was able to walk unaided. His cranial nerves were intact save for evidence of a right recurrent laryngeal nerve palsy on indirect laryngoscopy. There was a flicker of left biceps contraction and grade $2 / 5$ power in the deltoids and adductors of the shoulders, but otherwise total paralysis of the upper limbs. Marked tenderness was noted over each brachial plexus, but there was neither muscle tenderness nor fasciculation. 'Glove' loss of all sensory modalities was present up to the elbows bilaterally, with hypotonicity and areflexia. There was minimal hip flexor weakness and absent left knee and both ankle reflexes without sensory abnormality or calf tenderness. Bladder and bowel functions were unaffected and examination otherwise unremarkable. His blood pressure was $150 / 90 \mathrm{mmHg}$, and fundi normal. No skin lesions were apparent.

Investigations: The ESR was $30 \mathrm{~mm}$ in the first hour; Hb 14.1 g/dl; WBC 13.8 $\times 10^{\circ} / 1(2 \%$ eosinophils); repeated urinalyses proved negative; urea $12.0 \mathrm{mmol} / \mathrm{l}$, creatinine $86 \mu \mathrm{mol} / \mathrm{l}$, with normal electrolytes; bilirubin $11 \mu \mathrm{mol} / \mathrm{l}$, alkaline phosphatase $147 \mathrm{u}$./l, $\gamma$-glutamyl transpeptidase 270 i.u./l. Anti-nuclear factor, anti-smooth muscle antibody and rheumatoid factor were negative, as were the VDRL and TPHA in blood and cerebro-spinal fluid. (CSF) which was clear and under normal pressure, without a pleocytosis or increased protein concentration. His initial chest X-ray was normal, but films of cervical spine showed disc space narrowing at the C3/4 and C4/5 levels. Cervical myelography was normal. An isotope bone scan revealed several areas of increased uptake throughout the ribs. $\mathrm{Hb}_{\mathrm{s}} \mathrm{Ag}$ was not detected by radio-immunoassay. Nerve conduction studies showed an absence of nerve action potentials from the median nerves at wrist and elbow, with diminished amplitude in the ulnar nerves 
and slowing of conduction. Electromyography was unremarkable. Re-examination one month later showed profuse fibrillation in the forearm muscles with complete inexcitability of ulnar and median nerves bilaterally.

A diagnosis of atypical polymyalgia rheumatica had been made by his family doctor before admission and therapy commenced with prednisolone $40 \mathrm{mg}$ daily. This dose was increased to $80 \mathrm{mg}$ daily following admission to hospital. In spite of this, his condition progressively deteriorated with increasing paralysis and sensory loss in the upper limbs and the development of progressive weakness in the lower limbs, initially proximal but spreading distally. Breathing and swallowing difficulties arose, necessitating tracheostomy and intra-gastric feeding. Repeated chest radiography showed variable pneumonic shadowing, predominantly in the lower zones, unresponsive to antimicrobial therapy. Transient visual loss in his left eye occurred and was thought to be ischaemic or embolic in origin. He complained of increasingly severe pain in his thoracic cage in spite of analgesics and, as his bone scan was suggestive of metastatic disease, the unusual neuropathy was regarded as carcinomatous, predominantly affecting the upper limbs and right recurrent laryngeal nerve, but with increasing involvement of the lower limbs. Corticosteroid therapy was withdrawn and he died under sedation of a bronchopneumonia.

Post-mortem revealed widespread arteritis predominantly affecting medium and small arteries and arterioles. The lesions varied from those that were chronic in a healing phase to others that were acute, with fibrinoid necrosis of the media, polymorph infiltration and intraluminal thrombus formation. The lungs were conspicuously involved, showing extensive areas of necrosis with superadded bronchopneumonia, and associated venous involvement. Granulomata were not present in the bronchopulmonary tree or elsewhere. The brachial plexus, median, ulnar, sciatic and vagus nerves bilaterally and the right recurrent laryngeal nerve showed arteritis of the epineurial vessels. Several posterior root ganglia were involved but the central nervous system was unaffected. Scattered arteritic lesions were present in the kidneys, gut, spleen and adrenals. There was pronounced fatty change in the liver with large numbers of polymorphs within the sinusoids, consistent with a bacteraemia, but no arteritic lesions were identified. The appearances were regarded as typical of polyarteritis nodosa. The bony lesions seen on isotope scanning represented fibrous dysplasia. No evidence of primary or metastatic tumour was found.

\section{Discussion}

Peripheral neuropathic involvement occurs in up to $50 \%$ of patients with polyarteritis nodosa (Goetz, 1980), often appearing early in the course of the ill- $\frac{3}{8}$ ness and dominating the clinical picture. Neuro- $\varrho$ logical symptoms and signs may indeed provide the $\bar{c}$ major clinical evidence for the eventual diagnosis. $\overrightarrow{\vec{F}}$ In one series of 114 patients with proved polyar $-\overrightarrow{0}$ teritis nodosa (Ford and Siekert, 1965), 68\% had a $\frac{\bar{\sigma}}{\circ}$ peripheral neuropathy, of which group $58 \%$ had $\overline{\text {. }}$ mononeuritis multiplex, $16 \%$ a polyneuropathy and $\vec{\widetilde{ }}$ $26 \%$ a mixed pattern. Symmetrical polyneuropathy predominantly affecting the lower limbs, mostes marked distally and associated with muscle pain and $\overrightarrow{0}$ tenderness is well described (Goetz, 1980). Brachial $\overrightarrow{-}$ and lumbo-sacral plexopathies have been reported, $\vec{\sigma}$ but are so rare (Goetz, 1980) that it seemed worth- $\frac{0}{8}$ while describing a further case.

This patient's progressive symmetrical bilateral brachial plexopathy, with involvement of the right ${ }^{\infty}$ recurrent largyneal nerve, raised the possibility of an 20 atypical Guillain-Barré syndrome which may present ${ }_{\omega}^{\bullet}$ with upper limb motor involvement and loss of $\vec{\exists}$ deep touch, vibration and proprioception before응 spreading to involve laryngeal, palatal and pharyn- geal functions or, later, the lower limbs. The course $\bar{z}$ of this patient's illness and his normal CSF biochemistry were against this diagnosis. A pure $\vec{A}$ ascending motor polyneuropathy, progressing respiratory and swallowing difficulties, with pre sumed but mistaken diagnosis of Guillain-Barce

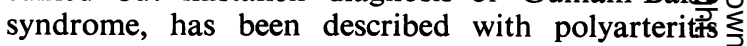
nodosa (Goetz, 1980) but is a rare presentation. Sensorimotor polyneuropathy is one of the recognized carcinomatous neuropathies and may be $\propto$ indistinguishable from the Guillain-Barré syndrome, $\overrightarrow{\vec{O}}$ save that sensory symptoms usually dominate the $\overline{3}$ clinical picture (Bruyn, 1979). In view of this patient's radio-isotope bone scan report, and faced with progressive clinical deterioration in spite of adequate corticosteroid therapy, the authors re- 3 garded carcinomatous polyneuropathy as a likely explanation of the picture and withdrew therapy. In retrospect, his broncho-pulmonary symptoms and 0 radiographic signs, together with the painful peripheral neuropathic involvement and retinal $\frac{\text { o }}{5}$ ischaemic episode were attributable to the inexorable $\rightarrow$ progress of his polyarteritis nodosa.

The post-mortem findings of severe pulmonary $N$ involvement raise the possibility of Wegener's granulomatosis, but the absence of respiratory tract $N$ granulomata and minimal renal involvement with- $\underset{\omega}{ }$ out granuloma formation is against this diagnosis. Attempts have been made artificially to distinguish 6 'classic' polyarteritis nodosa, in which there is spar- $\overparen{\varnothing}$ ing of the lungs, from allergic angiitis granulomatosis $\stackrel{\mathcal{P}}{+}$ where broncho-pulmonary involvement with pre- $T$ ceding asthma or bronchitis and a blood eosino- $\frac{\vec{D}}{\vec{D}}$ philia dominate the picture and granulomata are $\stackrel{\mathbb{Q}}{\stackrel{\oplus}{Q}}$ 
found in relation to the arteritic lesions (Austen, 1979; Churg and Strauss, 1951). This patient had intermittent wheeze and breathlessness for some months before admission, but the absence of a blood eosinophilia, together with the demonstration of widespread typical polyarteric lesions at postmortem, particularly in the lungs, highlights the difficulty in attempting artificially to differentiate ends of a spectrum in this rare and confusing disease. The unpredictable response of polyarteritis nodosa to corticosteroid therapy and its occurrence without obvious precipitant in the elderly is re-emphasized. The diagnosis should be suspected where atypical neurological syndromes arise in the absence of tumour or other obvious association and nerve biopsy should be considered at an early stage in the investigation of the patient.

\section{References}

Austen, K.F. (1979) CECIL Textbook of Medicine 15th edn, p. 180. Beeson, McDermott and Wyngaarden; W.B. Saunders Co., Philadelphia, London, Toronto.

BRUYN, G.W. (1979) Carcinomatous polyneuropathy. In: Handbook of Clinical Neurology, Vol. 38, p. 679. (Ed. by Vinken, P.J. \& Bruyn, G.W.) Elsevier, Amsterdam.

Churg, J. \& Strauss, L. (1951) Allergic granulomatosis, Allergic angiitis and peri-arteritis nodosa. American Journal of Pathology, 27, 277.

Ford, R.G. \& SiekeRT, R.G. (1965) Central nervous system manifestations of peri-arteritis nodosa. Neurology, 15, 114. Goetz, C.G. (1980) Polyarteritis nodosa. In: Handbook of Clinical Neurology, Vol. 39. (Ed. by Vinken, P.J. \& Bruyn, G.W.) p. 295. Elsevier, Amsterdam. 